\title{
Das Potential zwischen Nukleonen
}

\author{
Von Gernot Eder \\ Aus dem Max-Planck-Institut für Physik, Göttingen \\ (Z. Naturforschg. 9 a, 565-572 [1954]; eingegangen am 17. April 1954)
}

\begin{abstract}
Unter Voraussetzung der ladungssymmetrischen, pseudoskalaren Mesontheorie werden die nichtadiabatischen Korrekturen zur zweiten und vierten störungstheoretischen Näherung durch die Berücksichtigung der Nukleonenrückstöße berechnet. Es ergibt sich ein stark anziehendes Potential vierter Ordnung. Fragen der Spin-Bahn-Kopplung und der Absättigung der Kernkräfte werden behandelt.
\end{abstract}

$D^{i}$ ie Anwendung eines Störungsverfahrens bei der mesontheoretischen Berechnung des Potentials zwischen Nukleonen ist wegen der Größe der $\mathrm{Nu}$ kleon-Pion-Kopplungskonstante eine Methode, deren Konvergenz zweifelhaft erscheint. Allerdings ist die Reichweite der Potentialanteile, welche durch den Austausch von $n$ virtuellen Pionen entstehen, proportional $n^{-1}$. Die niederen Näherungen in der Kopplungskonstante $f$ werden daher das Verhalten des Potentials für größere Entfernungen gut wiedergeben; für kleine Abstände dominiert der Beitrag höherer Näherungen in $f$ und der Anteil der schweren Mesonen. Levy ${ }^{1}$ repräsentiert diese Beiträge durch einen phänomenologisch angenommenen Abstoßungsradius $r_{\mathrm{c}}$, wobei das Potential für $r<r_{\mathrm{c}}$ positiv unendlich sein soll. $r_{\mathrm{c}}$ wird dann so bestimmt, daß dadurch die niederenergetischen Daten am besten be-

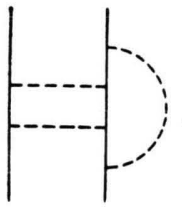

Abb. 1. LambshiftTerm. friedigt werden. Eine Schwierigkeit bei der Potentialberechnung für $r>r_{\mathrm{c}}$ bilden jene Terme, bei denen $m$ Pionen nicht ausgetauscht, sondern vom emittierenden Nukleon wieder absorbiert werden (Abb. 1). Diese Terme ergeben ein Potential, dessen Reichweite nur durch die Anzahl der ausgetauschten Pionen $(n-m)$ bestimmt ist, obwohl es sich um einen Beitrag der Ordnung $f^{n}$ handelt. Es werden also die größten Potentialbeiträge der Ordnung $f^{n-m}$ mit neuen Konstanten zu multiplizieren sein. We t z e $^{2}$ hat den Beitrag solcher LambshiftTerme nach der Methode der starken Kopplung in einer statischen Näherung $\left(\bar{\psi} \psi(\mathfrak{x}) \rightarrow \sum_{s} \delta\left(\mathfrak{x}-\mathfrak{x}_{s}\right)\right)$ berechnet. Da die Vernachlässigung der Nukleonenrückstöße die Beiträge der hochenergetischen virtuellen Pionen verfälscht, so werden bei ihm diese durch einen ad hoc eingeführten Grenzimpuls $p_{0}$

1 M. M. Levy, Phys. Rev. 88, 725 [1952].

${ }^{2}$ G. Wentzel, Phys. Rev. 86, 806 [1952]. abgeschnitten. Dadurch wird z. B. der Zweipaarterm vierter Ordnung $V_{4}^{\mathrm{a}}$ [vgl. (2)] reduziert auf

$$
V_{4}^{\mathrm{a}}\left(1+\frac{p_{0}}{M} \frac{f^{2}}{4 \pi}\right)^{-2}
$$

( $M$ Nukleonenmasse). Da Wentzel diesen Grenzimpuls in der Größenordnung von $M$ annimmt, so würden dadurch die Beiträge der Zweipaarterme sehr stark verkleinert werden. Allerdings läßt eine Verallgemeinerung der im folgenden verwendeten Methode vermuten, daß die Berücksichtigung der Nukleonenrückstöße einen so starken Abfall der Beiträge höherer Impulse mit sich bringt, daß man $p_{0} \ll M$ annehmen kann. Außerdem hat Jastrow ${ }^{3}$ gezeigt, daß die Deuterondaten gegen eine Variation von $V(\alpha, f)=V_{2}+V_{4}$ nach $\alpha$ durch eine leichte Änderung von $f$ unempfindlich gemacht werden können $\left[\alpha=1 \rightarrow \alpha=1 / 4\right.$ entspricht $\left.\left(f^{2} / 4 \pi\right)=10 \rightarrow\left(f^{2} / 4 \pi\right)=16\right]$. Aus diesen Gründen erscheint es doch wahrscheinlich, daß die erwähnten Übergänge keine zu großen Beiträge liefern. Wir werden uns daher im folgenden auf die von Klein ${ }^{4}$ behandelten Terme beschränken.

\section{Das adiabatische Potential}

Die störungstheoretische Berechnung des Potentials zwischen zwei Nukleonen wurde von Lev ${ }^{1}$ bzw. Klein ${ }^{4}$ nach der Methode von Tamm und Dancoff bzw. Bethe und Salpeter in zweiter und vierter störungstheoretischer Näherung durchgeführt. Levy hat in adiabatischer Näherung, d. h. bei Vernachlässigung der Nukleonenrückstöße ein Potential gefunden, welches mit $\left(f^{2} / 4 \pi\right)=10$ die Deuterondaten relativ gut befriedigt:

$$
\begin{aligned}
V_{2}(x)^{\mathrm{ad}}= & \left(\overrightarrow{\tau_{\mathrm{a}}} \cdot \overrightarrow{\tau_{\mathrm{b}}}\right)\left(\frac{f^{2}}{4 \pi}\right)(m / 2 M)^{2} m \\
& \cdot\left\{\frac{1}{3}\left(\overrightarrow{\sigma_{\mathrm{a}}} \cdot \overrightarrow{\sigma_{\mathrm{b}}}\right)+\left(\frac{1}{3}+\frac{1}{x}+\frac{1}{x^{2}}\right) S_{12}\right\} e^{-x} / x
\end{aligned}
$$

${ }^{3}$ R. Jastrow, Phys. Rev. 91, 749 [1953].

${ }^{4}$ A. Klein, Phys. Rev. 90, 1101 [1953]. 
$V_{4}^{\mathrm{a}}(x)^{\mathrm{ad}}=-3\left(f^{2} / 4 \pi\right)^{2}(m / 2 M)^{2} m(2 / \pi) K_{1}(2 x) / x^{2}$,

$V_{4}^{\mathrm{b}}(x)^{\mathrm{ad}}=-3\left(f^{2} / 4 \pi\right)^{2}(m / 2 M)^{3} m\left[(2 / \pi) K_{1}(x)\right]^{2} / x^{2}$,

wobei $x=m r$ und $m$ die Pionmasse bedeutet. Die Beiträge $V_{4}^{\mathrm{a}}$ und $V_{4}^{\mathrm{b}}$ kommen von solchen Übergängen, bei welchen jedes Nukleon einen Zwischenzustand durchläuft. Dieses Levysche Potential wurde von Lüders ${ }^{5}$ und Klein ${ }^{4}$ korrigiert: Entwickelt man die Energienenner jener Terme, die $V_{4}{ }^{a}$ ergeben, so wird durch das erste Entwicklungsglied gerade $V_{4}^{\mathrm{b}}$ kompensiert; durch die Einpaarterme entsteht noch ein zusätzliches abstoßendes Potential:

$V_{4}^{\prime}(x)=6\left(f^{2} / 4 \pi\right)^{2}(m / 2 M)^{3} m(1+1 / x)^{2} e^{-2 x} / x^{2}$.

Berücksichtigt man noch das Potential, welches von solchen Übergängen herrührt, bei welchen keine Nukleonenpaare in den Zwischenzuständen vorhanden sind, so erhalten wir zusätzlich:

$V_{4}^{\prime \prime}(x)=\left(f^{2} / 4 \pi\right)^{2}(m / 2 M)^{4} m$

$\cdot\left\{\left(\overrightarrow{\tau_{\mathrm{a}}} \cdot \overrightarrow{\tau_{\mathrm{b}}}\right) U_{\tau}(x)+\left(\overrightarrow{\sigma_{\mathrm{a}}} \cdot \overrightarrow{\sigma_{\mathrm{b}}}\right) U_{\sigma}(x)+S_{12} U_{T}(x)\right\}$,

wo $U_{\tau}, U_{\sigma}$ und $U_{T}$ bei Nishijima ${ }^{6}$ und Klein ${ }^{4}$ gegeben sind (vgl. auch Brueckner und Watson ${ }^{7}$ ). Durch $V_{4}^{\prime}$ wird das Potential vierter Ordnung so stark verändert, daß es die Deuterondaten nicht mehr befriedigen kann.

Die Korrekturen der Le v y schen Potentiale durch K le in weisen allerdings die Schwierigkeit auf, daß sie auf einer fraglichen Entwicklung der Energienenner beruhen: So wird z. B. für den Energienenner $\left(2 M+\omega_{1}\right)^{-1}\left(2 M+\omega_{2}\right)^{-1}$ die Entwicklung

$$
(2 M)^{-2} \sum_{n=0}^{\infty}\left(-\frac{\omega_{1}+\omega_{2}}{2 M}-\frac{\omega_{1} \omega_{2}}{(2 M)^{2}}\right)^{n} .
$$

verwendet und nach dem ersten Glied abgebrochen $\left(\omega_{i}{ }^{2}=m^{2}+\mathfrak{f}_{i}{ }^{2}\right)$. $(6)$ ist nur für $\omega_{1}+\omega_{2}+\omega_{1} \omega_{2} / 2 M$ $<2 M$ konvergent; da aber über den ganzen $\mathfrak{f}_{1}$ und $\mathfrak{f}_{2}$-Raum integriert wird, so erscheint die Verwendbarkeit von (6) sehr fraglich. So würde z. B. das Entwicklungsglied $\sim(2 M)^{-5}\left(\omega_{1}+\omega_{2}\right) \omega_{1} \omega_{2}$ ein Potential der Form $3 f^{4}(m / 2 M)^{5} m[\delta(m r)]^{2}$ liefern, während die höheren Glieder noch stärker divergieren. Mit einer Entwicklung des Integranden nach Potenzen von $(k / 2 M)$ fällt auch die Möglichkeit, das

\footnotetext{
${ }^{5}$ G. Lüders, Unveröff. Ber. d. Theor. Studiengruppe des Europ. Rats f. kernphys. Forschung (CERN), Kopenhagen, Dez. 1952.
}

${ }^{6}$ K. Nishijima, Progr. Theor. Phys. 6, 911 [1951]. adiabatische Potential als eine Potenzreihe nach $(m / 2 M)$ darzustellen. Auf einer analogen Entwicklung beruhen die Potentialkorrekturen von Sato ${ }^{8}$. Wir glauben also, daß die Kleinschen Korrekturen in ihrer bisherigen Form nicht gerechtfertigt werden können und unternehmen im folgenden eine Neuberechnung dieser Potentiale unter wesentlich genauerer Berücksichtigung der Nukleonenrückstöße.

\section{Das nichtadiabatische Potential}

Die einzeitige Bethe-Salpeter-Gleichung führt für die Wellenfunktion $\psi(\mathfrak{r})$ zweier Nukleonen a und $\mathbf{b}$ $\left(\mathfrak{r}=\mathfrak{r}_{b}-r_{a}\right)$ auf eine Gleichung der Form ${ }^{4}$ :

$$
\left(\frac{1}{2 M^{*}} \Delta+\varepsilon\right) \psi(\mathfrak{r})=\int \mathrm{d}^{3} \mathfrak{r}^{\prime} V\left(\mathfrak{r}, \mathfrak{r}^{\prime}\right) \psi\left(\mathfrak{r}^{\prime}\right),
$$

wo $M^{*}=M_{\mathrm{a}} M_{\mathrm{b}} /\left(M_{\mathrm{a}}+M_{\mathrm{b}}\right)$ und $\varepsilon=W-2 M$ die Bindungsenergie bedeuten. Das nichtlokale Potential $V\left(\mathfrak{r}, \mathfrak{r}^{\prime}\right)$ ist in zweiter Ordnung $\left(\sim f^{2}\right)$ gegeben durch $V_{2}\left(\mathfrak{r}, \mathfrak{r}^{\prime}\right)=-f^{2} \int \frac{\mathrm{d}^{3} \mathfrak{p}}{(2 \pi)^{3}} \frac{\mathrm{d}^{3} \mathfrak{p}^{\prime}}{(2 \pi)^{3}} \cdot e^{i\left(\mathfrak{p r}-\mathfrak{p}^{\prime} \mathfrak{r}^{\prime}\right)}$

$\cdot<\mathfrak{p},-\mathfrak{p}\left|\frac{\left.\left(\beta \gamma_{5} \tau_{i}\right)^{\mathrm{a}} \beta \gamma_{5}-\tau_{i}\right)^{\mathrm{b}}}{\omega_{p p^{\prime}}\left(\omega_{p}-p^{\prime}+E_{p}+E_{p^{\prime}}-W\right)}\right| \mathfrak{p}^{\prime},-\mathfrak{p}^{\prime}>$

$E_{p}^{2}=M+p^{2}$. Levy und Klein erhalten aus (8) ein lokales Potential, indem sie $E_{p}=E_{p^{\prime}}=M, W=2 M$ setzen. Diese Näherung führt aber zu einem falschen Ergebnis, da der Integrand von (8) über den ganzen Raum von $\mathfrak{p}$ und $\mathfrak{p}^{\prime}$ integriert wird. Es soll nun im folgenden gezeigt werden, daß dieser Fehler wohl für $m r \gg 1$ keine Rolle mehr spielt, sich aber bereits stark auswirkt für Entfernungen $m r \approx 1$, welche für das Deuteron entscheidend sind.

Entwickeln wir $\psi\left(\mathfrak{r}^{\prime}\right)$ in der Form

$$
\psi\left(\mathfrak{r}^{\prime}\right)=\psi(\mathfrak{r})+\left(\mathfrak{r}^{\prime}-\mathfrak{r}, \Delta \psi\right)+\ldots
$$

und setzen dies in (7) ein, so ergibt das erste Glied von (9) ein statisches Potential; die höheren Glieder geben wegen $-i \Delta \psi=\mathfrak{p} \psi$ geschwindigkeitsabhängige Potentiale (unter anderem eine Spin-BahnKopplung). Wir interessieren uns zunächst nur für das statische Potential: Setzen wir das erste Glied von (9) in (7) ein, so folgt in zweiter störungstheoretischer Näherung:

$$
\left(\frac{1}{2 M^{*}} \Delta+\varepsilon\right) \psi(\mathfrak{r})=V_{2}(\mathfrak{r}) \psi(\mathfrak{r}),
$$

${ }^{7}$ K. A. Brueckner u. K. M. Watson, Phys. Rev. 92, 1023 [1953].

${ }^{8}$ I. Sato, Progr. Theor. Phys. 10, 323 [1953]. 
wo

$$
\begin{aligned}
& V_{2}(\mathfrak{r})=\int \mathrm{d}^{3} \mathfrak{r}^{\prime} V_{2}\left(\mathfrak{r}, \mathfrak{r}^{\prime}\right) \\
& =-\int \frac{\mathrm{d}^{3} \mathfrak{p}}{(2 \pi)^{3}} e^{i \mathfrak{p r}}<\mathfrak{p},-\mathfrak{p}|\ldots| 0,0>.
\end{aligned}
$$

Mit $< \pm \mathfrak{p}\left|\beta \gamma_{5}\right| 0>= \pm\left[2 E_{p}\left(M+E_{p}\right)\right]^{-1 / 2}(\vec{\sigma} \cdot \mathfrak{p})$ wird

$$
V_{2}(\mathrm{r})=(f / 2 M)^{2}\left(\overrightarrow{\tau_{\mathrm{a}}} \cdot \overrightarrow{\tau_{\mathrm{b}}}\right)\left(\overrightarrow{\sigma_{\mathrm{a}}} \cdot \Delta\right)\left(\overrightarrow{\sigma_{\mathrm{b}}} \cdot \Delta\right) Y(r),
$$

wo $\quad Y(r)=\int \frac{\mathrm{d}^{3} \mathfrak{p}}{(2 \pi)^{3}} \frac{e^{i \mathfrak{p r}}}{\omega_{p}^{2}} \varphi(p)$;

dabei ist

$\varphi(p)=2 M^{2}\left\{E_{p}\left(M+E_{p}\right)\left(1+\frac{E_{p}-M-\varepsilon}{\omega_{p}}\right)\right\}^{-1}$.

In adiabatischer Näherung wird $\varphi(p)^{\text {ad }}=1$ gesetzt, während das exakte $\varphi(p)$ ungefähr wie

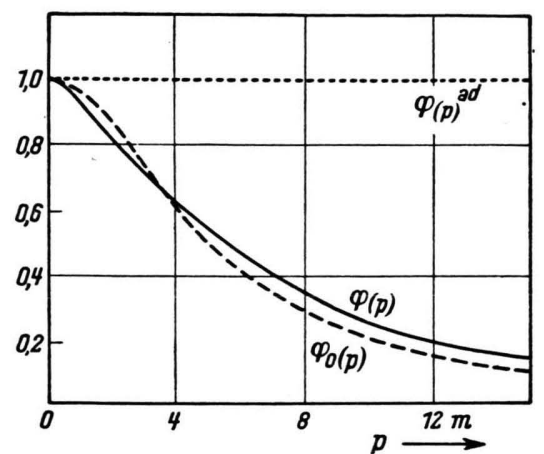

Abb. 2. Der Einfluß der Nukleonenrückstöße beim Potential 2. Ordnung.

$$
\varphi_{0}(p)=25 m^{2}\left(25 m^{2}+p^{2}\right)^{-1}
$$

verläuft (Abb. 2). Setzen wir (13) in (12) und (12) in (11) ein, so erhalten wir:

$$
\begin{aligned}
V_{2}(x)= & \frac{25}{24}\left(\frac{f^{2}}{4 \pi}\right)\left(\frac{m}{2 M}\right)^{2} m\left(\overrightarrow{\tau_{\mathrm{a}}} \cdot \overrightarrow{\tau_{\mathrm{b}}}\right) \\
& \cdot\left\{\frac{1}{3}\left(\overrightarrow{\sigma_{\mathrm{a}}} \cdot \overrightarrow{\sigma_{\mathrm{b}}}\right)\left(1-25 e^{-4 x}\right)+\left[\frac{1}{3}+\frac{1}{x}+\frac{1}{x^{2}}\right.\right. \\
& \left.\left.-\left(\frac{25}{3}+\frac{5}{x}+\frac{1}{x^{2}}\right) e^{-4 x}\right] S_{12}\right\} \frac{e^{-x}}{x}
\end{aligned}
$$

Das Kontaktpotential $\sim \delta(\mathfrak{r})$ fällt weg.

Für den ${ }^{3} S$-Zustand folgt aus (14) mit $\left(f^{2} / 4 \pi\right)=10$ und $m=141 \mathrm{MeV}$ :

$$
\begin{aligned}
V_{2}\left(x,{ }^{3} S\right) & =-8,18 \mathrm{MeV}\left\{1-25 e^{-4 x}+\left[1+\frac{3}{x}+\frac{3}{x^{2}}\right.\right. \\
& \left.\left.-\left(25+\frac{15}{x}+\frac{3}{x^{2}}\right) e^{-4 x}\right] S_{12}\right\} \frac{e^{-x}}{x} .
\end{aligned}
$$

Die Zentralkraft wird also für $m r<0,8$ abstoßend, während die Tensorkraft immer negativ bleibt; doch wird ihr Betrag für kleine Radien stark heruntergedrückt (für $x=m r=0,4$ beträgt sie $42 \%$ ihres adiabatischen Wertes). Für $m r \gg 1$ erhalten wir die adiabatische Näherung (1).

Die Potentialbeiträge vierter Ordnung unterscheiden wir entsprechend ihren Zwischenzuständen in Zweipaar-, Einpaar- und in solche Terme, welche keine virtuellen Paare enthalten. Die Zweipaarterme liefern das Potential $V_{4}{ }^{a}$ und $V_{4}{ }^{b}$. Es ist

$$
\begin{aligned}
V_{4}^{\mathrm{a}}(r)=-\frac{3}{4} f^{4} & \left.\int \frac{\mathrm{d}^{3} \mathfrak{f}_{1}}{(2 \pi)^{3}} \frac{\mathrm{d}^{3} \mathfrak{f}_{2}}{(2 \pi)^{3}} \frac{e^{i\left(\mathfrak{f}_{1}+\mathfrak{f}_{2}\right) \mathrm{r}}}{\omega_{1} \omega_{2}}\left\{\frac{<\mathfrak{f}_{1}+\mathfrak{f}_{2}\left|\beta \gamma_{1} \Lambda\left(\mathfrak{f}_{2}\right) \beta \gamma_{5}\right| 0>\mathrm{a}}{\left(\omega_{1}+\omega_{2}+E_{12}-M-\varepsilon\right)} \cdot \frac{<-\mathfrak{f}_{12}-\mathfrak{f}_{2}\left|\beta \gamma_{5} \Lambda\left(-\mathfrak{f}_{1}\right) \beta \gamma_{5}\right| 0>\mathrm{b}}{E_{12}+E_{1}+\omega_{2}}\right]+1 \rightleftarrows 2\right\}= \\
\cdot & {\left[\frac{<-\mathfrak{f}_{1}-\mathfrak{f}_{2}\left|\beta \gamma_{5} \Lambda\left(-\mathfrak{f}_{2}\right) \beta \gamma_{5}\right| 0>^{\mathrm{b}}}{E_{12}+E_{2}+\omega_{1}}+\frac{<-f^{2}}{4 \pi^{2} M r}\right)^{2} \int_{0}^{\infty} \mathrm{d} k_{1} \int_{0}^{\infty} \mathrm{d} k_{2} \omega_{1}^{-1} \omega_{2}^{-1} \varphi\left(k_{1}, k_{2} ; \overline{E_{12}}\right) \sin \left(k_{1} r\right) \sin \left(k_{2} r\right) k_{1} k_{2}, }
\end{aligned}
$$

wo $\varphi\left(k_{1}, k_{2} ; \bar{E}_{12}\right)=\frac{M^{2}}{8} \cdot \frac{\left(1+M / \overline{E_{12}}\right)\left(1+M / E_{2}\right)}{\left(\omega_{1}+\omega_{2}+\overline{E_{12}}-M-\varepsilon\right)\left(M+\overline{E_{12}}+\omega_{2}\right)}\left(\frac{1+M / E_{2}}{\overline{E_{12}}+E_{2}+\omega_{1}}+\frac{1+M / E_{1}}{\overline{E_{12}}+E_{1}+\omega_{2}}\right)+1 \rightleftarrows 2$, $E_{i}=+\left[M^{2}+\mathfrak{l}_{i}{ }^{2}\right]^{1 / 2}, \quad E_{12}=+\left[M^{2}+\left(\mathfrak{f}_{1}+\mathfrak{f}_{2}\right)^{2}\right]^{1 / 2}$.

$\bar{E}_{12}$ ist ein Mittelwert von $E_{12}$ zwischen dem Maximalwert $E_{\mathrm{m}}=+\left[M^{2}+\left(\left|\mathfrak{f}_{1}\right|+\left|\mathfrak{f}_{2}\right|\right)^{2}\right]^{1 / 2}$ und dem Minimalwert $M$. Dieser Mittelwert wird eingeführt, um komplizierte Winkelintegrationen zu vermeiden. Im adiabatischen Grenzfall wird

$$
\varphi\left(k_{1}, k_{2}\right)^{\mathrm{ad}}=\left(\omega_{1}+\omega_{2}\right)^{-1} .
$$

Am Rande des Integrationsbereiches $\left(k_{1}=0\right.$ bzw. $\left.k_{2}=0\right)$ ist $E_{12}=E_{2}$ bzw. $E_{1}, \varphi(0, k)=\varphi(k, 0)$ daher eindeutig bestimmt (Abb. 3). Je weiter wir uns aber der Geraden $k_{1}=k_{2}=k$ nähern, desto größer ist der Bereich, in dem $\bar{E}_{12}$ liegen kann. Allerdings weicht sogar $\varphi\left(k, k ; E_{\mathrm{m}}\right)$ nicht zu stark von $\varphi(k, k ; M)$ ab (Abb. 4), so daß wir einen guten Näherungsaus- 
$\operatorname{druck} \varphi_{0}\left(k_{1}, k_{2}\right)$ erhalten, wenn wir diesen so wählen, daß $\varphi_{0}(k, 0)=\varphi_{0}(0, k) \approx \varphi(k, 0)=\varphi(0, k)$ und daß $\varphi_{0}(k, k)$ zwischen $\varphi\left(K, k ; E_{\mathrm{m}}\right)$ und $\varphi(k, k ; M)$ liegt (Abb. 3) und (Abb. 4).

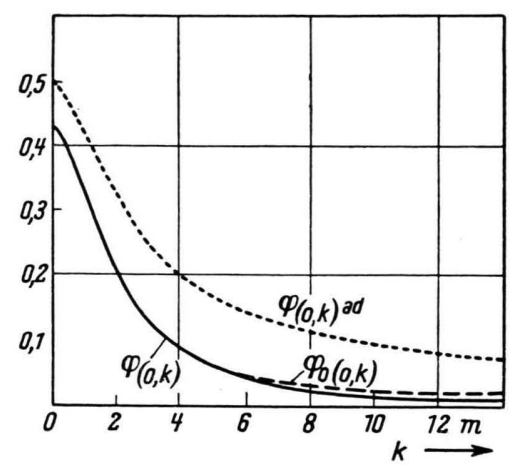

Abb. 3. Der Einfluß der Nukleonenrückstöße bei $V_{4}$ a am Rand des Integrationsgebietes.

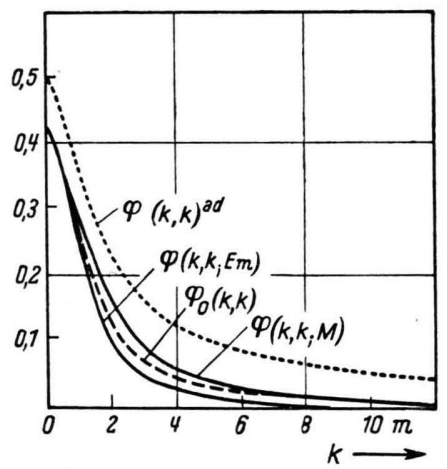

Abb. 4. Der Einfluß der Nukleonenrückstöße bei $V_{4}{ }^{a}$ für $k_{1}=k_{2}=k$.

Dabei gilt:

$\varphi_{0}\left(k_{1}, k_{2}\right)=\frac{0,0966}{\omega_{1}+\omega_{2}}+\frac{2,201}{\left(\omega_{1}+\omega_{2}\right)^{2}}-\frac{0,0383}{\omega_{1} \omega_{2}}-\frac{0,128}{\omega_{1}^{2} \omega_{2}^{2}}$.

(17) gibt in (16) eingesetzt:

$$
\begin{aligned}
V_{4}^{\mathrm{a}}(x) & =-3\left(\frac{f^{2}}{4 \pi} \frac{m}{2 M}\right)^{2} m \\
& \cdot\left\{\left[0,0966 \frac{2}{\pi} K_{1}(2 x)-0,0383 e^{-2 x}\right] x^{-2}\right. \\
& \left.+1,101\left[\frac{2}{\pi} K_{1}(x)\right]^{2}-1,128\left[\frac{2}{\pi} K_{0}(x)\right]^{2}\right\} .
\end{aligned}
$$

$V_{4}{ }^{\text {a }}$ steigt von $47 \%$ bei $x=0,4$ auf $137 \%$ seines adiabatischen Wertes (2) bei $x=1,8$.

Entsprechend gilt:

$V_{4}^{\mathrm{b}}(x)=-3\left(\frac{f^{2}}{4 \pi^{2} M r}\right)^{2}$

$\cdot \int_{0}^{\infty} \mathrm{d} k_{1} \int_{0}^{\infty} \mathrm{d} k_{2} \frac{k_{1} k_{2}}{\omega_{1} \omega_{2}} \varphi\left(k_{1}, k_{2}\right) \sin \left(k_{1} r\right) \sin \left(k_{2} r\right)$, wo $\varphi\left(k_{1}, k_{2}\right)=\frac{M^{3}}{2} \frac{\left(1+M \bar{E} / /_{12}\right)\left(1+M / E_{2}\right)}{\left(\bar{E}_{12}+E_{2}+\omega_{1}\right)\left(E_{2}+M+\omega_{2}\right)}$

$$
\cdot\left\{\frac{1+M / E_{2}}{W+2 E_{2}}+\frac{1+M / E_{1}}{W+E_{1}+E_{2}+\omega_{1}+\omega_{2}}\right\}+1 \rightleftarrows 2 .
$$

Gehen wir genau so wie bei $V_{4}{ }^{a}$ vor, so erhalten wir mit

$$
\begin{aligned}
\varphi\left(k_{1}, k_{2}\right) \approx \varphi_{0}\left(k_{1}, k_{2}\right) \\
=\frac{4,755 \omega_{1} \omega_{2} m^{2}}{\left(3 m^{2}+k_{1}{ }^{2}\right)\left(3 m^{2}+k_{2}{ }^{2}\right)}+\frac{0,6145 m}{\omega_{1}+\omega_{2}}, \quad \\
\left(\varphi\left(k_{1}, k_{2}\right)^{\mathrm{ad}}=1\right): \\
V_{4}^{\mathrm{b}}(x)=-3\left(f^{2} / 4 \pi\right)^{2}(m / 2 M)^{3} m \\
\cdot\left\{4,755 e^{-2 \sqrt{3} x}+0,6145(2 / \pi) k_{1}(2 x)\right\} / x^{2} .
\end{aligned}
$$

$V_{4}{ }^{\mathrm{b}}(x)$ steigt von $79 \%$ seines adiabatischen Wertes bei $x=0,4$ auf $129 \%$ bei $x=1,8$.

Der Beitrag der Einpaarterme lautet:

$$
\begin{aligned}
& V_{4}^{\prime}(x)=-6 f^{4}(2 M)^{-3} \\
& \quad \cdot \int \frac{d^{3} f_{1}}{(2 \pi)^{3}} \frac{d^{3} \mathfrak{f}_{2}}{(2 \pi)^{3}} \cdot \frac{e^{i\left(\mathfrak{f}_{1}+\mathfrak{f}_{2}\right) \mathfrak{r}}}{\omega_{1}{ }^{2} \omega_{2}{ }^{2}} \varphi\left(k_{1}, k_{2}\right)\left(\mathfrak{f}_{1}, \mathfrak{f}_{2}\right) .
\end{aligned}
$$
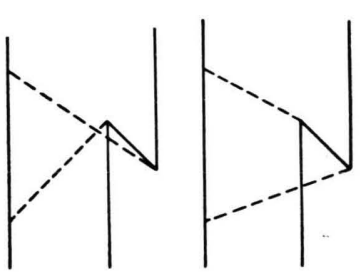

Abb. 5.

Einpaar-Übergänge.
In adiabatischer Näherung gilt $\varphi\left(k_{1}, k_{2}\right)^{\text {ad }}=1$. Um den Einfluß der bei Nukleonenrückstöße den Einpaartermen abzuschätzen, beschränken wir uns der Einfachheit halber auf Graphen der Form Abb. 5.

Damit ergibt sich

$$
\begin{gathered}
\varphi\left(k_{1}, k_{2}\right)=\frac{M^{3}}{2 E_{12}}\left\{\frac { E _ { 2 } + M } { 2 E _ { 2 } } \left[\left(E_{1}\left(1+\frac{E_{1}-M}{\omega_{1}}\right)\right.\right.\right. \\
\left.\cdot\left(1+\frac{E_{1}+E_{12}-2 M}{\omega_{2}}\right)\left(E_{1}+E_{2}+E_{12}-M\right)\right)^{-1} \\
+\left(E_{2}\left(1+\frac{E_{2}+E_{12}-2 M}{\omega_{1}}\right)\left(1+\frac{E_{2}-M}{\omega_{2}}\right)\right. \\
\left.\left.\left.\cdot\left(2 E_{2}+E_{12}-M\right)\right)^{-1}\right]+1 \rightleftarrows 2\right\}
\end{gathered}
$$

(es wurde $W=2 M$ gesetzt). Die numerische Durchrechnung zeigt, daß man mit einer Genauigkeit von ca. $5 \%$ für $\varphi$ den Näherungsausdruck

$$
\varphi_{0}\left(k_{1}, k_{2}\right)=81 m^{4} /\left[\left(9 m^{2}+k_{1}^{2}\right)\left(9 m^{2}+k_{2}^{2}\right)\right]
$$

verwenden kann. (22) gibt dann mit (23):

$$
V_{4}^{\prime}(x)=\varphi(x) V_{4}{ }^{\prime}(x)^{\mathrm{ad}},
$$

wo $\varphi(x)=(81 / 64)\left[1+(3 x+1) /(x+1) \cdot e^{-2 x}\right]^{2}$.

$\varphi(x)$ steigt von $11 \%$ bei $x=0,4$ auf $111 \%$ bei $x=1,8$. Durch $\varphi(x)(25)$ wird der Beitrag des abstoßenden Potentials $V_{4}{ }^{\prime}$ wesentlich heruntergedrückt. Der Ein- 
fluß der Energienenner ist also für $V_{2}$ und $V_{4}^{\prime}$ viel größer als für $V_{4}^{\mathrm{a}}$ und $V_{4}^{\mathrm{b}}$. Dies rührt daher, daß die Zähler der Integranden von $V_{2}$ bzw. $V_{4}{ }^{\prime}$ proportional $\left(\overrightarrow{\sigma_{\mathrm{a}}} \cdot \mathfrak{f}\right)\left(\overrightarrow{\sigma_{\mathrm{b}}} \cdot \mathfrak{f}\right)$ bzw. $\left(\mathfrak{f}_{1} \cdot \mathfrak{f}_{2}\right)$ sind, also erst für große $\mathfrak{f}$ einen entscheidenden Beitrag liefern. Für großes $\mathfrak{f}$ ist aber auch der Einfluß der Energienenner groß.

Diese Reduktion der Potentialbeiträge durch die Energienenner ist besonders groß bei den Beiträgen jener Übergänge, welche in den Zwisehenzuständen keine Nukleonenpaare enthalten. Betrachten wir z. B. den Zentralkraftanteil der Übergänge, Abb. 6, so lautet der entsprechende Potentialbeitrag

$$
\begin{aligned}
V^{\prime \prime}(x) & =-\frac{3 f^{4}}{(2 M)^{4}} \\
\cdot & \int \frac{\mathrm{d}^{3} \mathfrak{f}_{1}}{(2 \pi)^{3}} \frac{\mathrm{d}^{3} \mathfrak{f}_{2}}{(2 \pi)^{3}} \frac{e^{i\left(\mathfrak{f}_{1}+\mathfrak{f}_{2}\right) \mathrm{r}}}{\omega_{1}{ }^{2} \omega_{2}{ }^{2}}\left(\mathfrak{f}_{1} \mathfrak{f}_{2}\right)^{2} \varphi\left(k_{1}, k_{2}\right),
\end{aligned}
$$

wo $\varphi\left(k_{1}, k_{2}\right)=2 M^{3}$

$$
\begin{aligned}
& \cdot\left\{E_{12}\left(E_{12}+M\right)\left[1+\left(E_{12}+E_{2}-2 M\right) / \omega_{1}\right]\right. \\
& \left.\cdot\left(\omega_{1}+\omega_{2}+E_{12}-M\right) E_{2}\right\}^{-1} \\
& \cdot\left\{\left(M / E_{2}\right)\left[1+\left(E_{2}-M\right) / \omega_{2}\right]\right. \\
& \left.+\omega_{2}\left(M / E_{1}\right) /\left(\omega_{1}+E_{1}-M\right)\right\} .
\end{aligned}
$$

$\varphi\left(k_{1}, k_{2}\right)^{\text {ad }}=\omega_{1}^{-1}$. Mit $\varphi_{0}\left(k_{1}, k_{2}\right)=1,96 \cdot 3,24 m^{3}$ $\cdot\left(1,96 m^{2}+k_{1}^{2}\right)^{-1}\left(3,24 m^{2}+k^{2}\right)^{-1}$ wird $(26)$ :

$V^{\prime \prime}(x)=$

$-3\left(f^{2} / 4 \pi\right)^{2}(m / 2 M)^{4} m(1,96 \cdot 3,24 / 0,96 \cdot 2,24) x^{-6}$ $\cdot\left\{\left[x^{2}+2 x+2\right] e^{-x}-\left[(1,8 x)^{2}+2(1,8 x)+2\right] e^{-1,8 x}\right\}$ $\cdot\left\{\left[x^{2}+2 x+2\right] e^{-x}-\left[(1,4 x)^{2}+2(1,4 x)+2\right] e^{-1,4 x}\right\}$, während die adiabatische Näherung

$$
\begin{aligned}
V^{\prime \prime}(x)^{\mathrm{ad}}=-3\left(f^{2} / 4 \pi\right)^{2}(m / 2 M)^{4} m(2 / \pi) \\
\cdot\left[x k_{0}(x)+k_{1}(x)\right]\left(x^{2}+2 x+2\right) e^{-x} / x^{4}
\end{aligned}
$$

ergibt. Die nichtadiabatischen Korrekturen ergeben nach (27) für $x=0,4$ eine Reduktion auf $0,7 \%$ des adiabatischen Wertes (28); für $x=1,8$ beträgt die Reduktion noch $60 \%$. Für $\left(f^{2} / 4 \pi\right)=10$ steigt $(27)$ von $-1,3 \mathrm{MeV}$ bei $x=0,4$ auf $-0,03 \mathrm{MeV}$ bei $x=1,8 . V_{4}^{\prime \prime}(x)$ kann daher vernachlässigt werden.

Bei den Beiträgen sechster Ordnung ist die Reduktion durch die Nukleonenrückstöße ebenfalls sehr groß. Der Hauptterm lautet:

$$
\begin{array}{r}
V_{6}(x)=-4\left(f^{2} / 4 \pi\right)^{3}(2 M)^{-4}\left(\overrightarrow{\tau_{\mathrm{a}}} \cdot \overrightarrow{\tau_{\mathrm{b}}}\right)\left(2 \pi^{2}\right)^{-3} \\
\cdot \int \mathrm{d}^{3} \mathfrak{f}_{1} \mathrm{~d}^{3} \mathfrak{f}_{2} \mathrm{~d}^{3} \mathfrak{f}_{3}\left(\omega_{1} \omega_{2} \omega_{3}\right)^{-2} \\
\cdot e^{i\left(\mathfrak{f}_{1}+\mathfrak{f}_{2}+\mathfrak{f}_{3}\right) \mathrm{r}} \cdot \varphi\left(k_{1}, k_{2}, k_{3}\right) \cdot\left(\overrightarrow{\sigma_{\mathrm{a}}} \cdot \mathfrak{f}_{1}\right)\left(\overrightarrow{\sigma_{\mathrm{b}}} \cdot \mathfrak{f}_{2}\right) .
\end{array}
$$

Setzt $\operatorname{man} \varphi=\varphi^{\mathrm{ad}}=1$, so wird nach K lein:

$$
\begin{aligned}
V_{6}(x)^{\mathrm{ad}}= & \frac{4}{3}\left(f^{2} / 4 \pi\right)^{3}(m / 2 M)^{4} m\left(\overrightarrow{\tau_{\mathrm{a}}} \cdot \overrightarrow{\tau_{\mathrm{b}}}\right) \\
& \cdot\left[\left(\overrightarrow{\sigma_{\mathrm{a}}} \cdot \overrightarrow{\sigma_{\mathrm{b}}}\right)+S_{12}\right]\left(1+\frac{1}{x}\right)^{2} e^{-3 x} / x^{3} .
\end{aligned}
$$

Um den Einfluß der Nukleonenrückstöße abzuschätzen, betrachten wir den Graphen Abb. 7. Für diesen Übergang lautet $\varphi$ :

$$
\begin{array}{r}
\varphi\left(k_{1}, k_{2}, k_{3}\right)=M^{2}\left(1+M / E_{1}\right)\left(1+M / \bar{E}_{13}\right)\left(1+M / \bar{E}_{23}\right) \\
\cdot\left\{4 E_{3} \bar{E}_{123}\left[1+\left(E_{3}+\bar{E}_{13}-2 M\right) / \omega_{1}\right]\right. \\
\cdot\left[1+\left(\bar{E}_{123}+\bar{E}_{13}-2 M\right) / \omega_{2}\right]\left[1+\left(E_{3}-M\right) / \omega_{3}\right] \\
\cdot\left[\left(E_{3}+\bar{E}_{13}+\bar{E}_{123}+\bar{E}_{23}\right) /(2 M)-1\right] \\
\left.\cdot\left[\left(E_{1}+E_{3}+\bar{E}_{13}\right) / M-1\right]\right\}^{-1},
\end{array}
$$

wo $\bar{E}_{12}=+\left[M^{2}+\overline{\left(\mathfrak{f}_{1}+\mathfrak{f}_{2}\right)^{2}}\right]^{1 / 2}$ usw.,

$$
\bar{E}_{123}=+\left[M^{2}+\overline{\left(\mathfrak{f}_{1}+\mathfrak{f}_{2}+\mathfrak{f}_{3}\right)^{2}}\right]^{1 / 2} \text {. }
$$

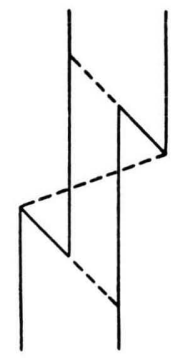

Abb. 7. Übergang sechster Ordnung.

Da $\varphi$ nur schwach von $k_{2}$ abhängt, können wir diese Abhängigkeit vernachlässigen. Für

$$
\varphi\left(k_{1}, k_{3}\right)=\varphi\left(k_{1}, 0, k_{3}\right)
$$

gehen wir beim Potential vierter Ordnung vor und erhalten als Näherungsausdruck für $\varphi$ :

$$
\begin{gathered}
\varphi_{0}\left(k_{1}, k_{2} \cdot k_{3}\right)=2,89 m^{2} \cdot 1,69 m^{2}\left(2,89 m^{2}+k_{1}^{2}\right)^{-1} \\
\cdot\left(1,69 m^{2}+k_{3}^{2}\right)^{-1} .
\end{gathered}
$$

Setzen wir (31) in (29) ein, so erhalten wir:

$$
V_{6}(x)=\varphi(x) V_{6}{ }^{\text {ad }}(x),
$$

$$
\text { wo } \varphi(x)=3,745\left[1-e^{-0,7 x}(1+1,7 x) /(1+x)\right]
$$

$$
\cdot\left[1-e^{-0,3 x}\right] \text {. }
$$

$\varphi(x)$ steigt von 0,04 bei $x=0,4$ auf 0,92 bei $x=1,8$. Für $\left(f^{2} / 4 \pi\right)=10$ steigt $V_{6}\left({ }^{1} S\right)$ von $-61,4 \mathrm{MeV}$ für $x=0,4$ auf $-0,03 \mathrm{MeV}$ bei $x=1,8$. Da die Berücksichtigung der Nukleonenrückstöße desto schwieriger wird, je mehr virtuelle Mesonen ausgetauscht werden, so wollen wir mit dem Potential sechster Ordnung diese Berechnungen abschließen. Addieren wir die Potentialbeiträge vierter Ordnung, so wird $V_{4}=V_{4}{ }^{\mathrm{a}}+V_{4}^{\mathrm{b}}+V_{4}^{\prime}$ ungefähr halb so groß wie das entsprechende Potential von Levy $\left(V_{4}^{\mathrm{a}}{ }^{\mathrm{ad}}+V_{4}^{\mathrm{b}}{ }^{\mathrm{ad}}\right)$. Dies spricht dafür, daß $\left(f^{2} / 4 \pi\right) \approx 10 \sqrt{2} \approx 14$ ange- 
nommen werden muß. Abb. 8 und 9 zeigen den Verlauf der Zentral- und Tensorkraft in S-Zuständen, wie er sich aus $V_{2}+V_{4}+V_{6}$ ergibt, für $\left(f^{2} / 4 \pi\right)=14$. Das Potential von Levy mit $\left(f^{2} / 4 \pi\right)=10$ ist zum Vergleich angegeben.

\section{Absättigung und Mehrkörperkräfte}

Wir untersuchen jetzt die Frage, ob man die Massendefekte der schwereren Atomkerne mit den von den $\pi$-Mesonen herrührenden Kräften allein -

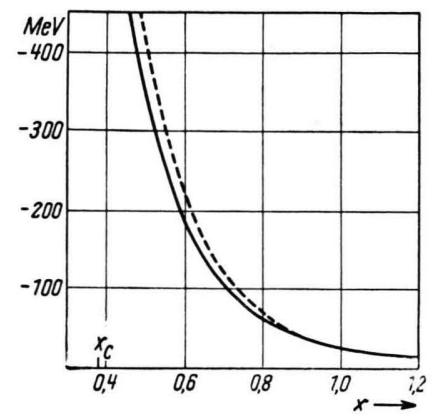

Abb. 8. Potential der Zentralkraft in $S$-Zuständen für Proton-Neutron-Wechselwirkung.

$\left(f^{2} / 4 \pi\right)=14 ;-\cdots$ Levy mit $\left(f^{2} / 4 \pi\right)=10$.

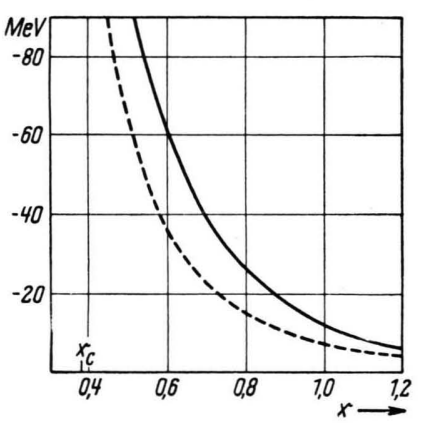

Abb. 9. Potential der Tensorkraft in $S$-Zuständen. $-\left(f^{2} / 4 \pi\right)=14 ;--$ Levy mit $\left(f^{2} / 4 \pi\right)=10$.

unter ganz summarischer Berücksichtigung der schwereren Mesonen durch den Grenzradius $r_{\mathrm{c}}$ deuten kann. Die Tatsache, daß die Absättigung der Kernkräfte nicht durch die Austauschwirkung der Zweikörperkräfte erklärt werden kann, weist auf den Einfluß von Mehrkörperkräften hin. Drell und Huang ${ }^{9}$ haben den Einfluß der Mehrkörperkräfte berechnet, wobei sie sich bei der $n$-Körperkraft auf jene Beiträge $2 n$-ter Ordnung in $f$ beschränken, welche die meisten Nukleonenpaare enthalten. Für

${ }^{9}$ S. D. Drell u. K. Huang, Phys. Rev. 91, 1527 [1953]. die Zweikörperkraft setzen sie das Potential $V_{4}{ }^{\text {a ad }}$ mit der Levyschen Kopplungskonstante $\left(f^{2} / \mathbf{4} \pi\right)=10$ als richtig voraus. Es soll nun untersucht werden, wie sich die Absättigungsverhältnisse für unsere modifizierten Zweikörperpotentiale ändern.

Nehmen wir für unser Potential wieder einen Abstoßungsradius $r_{\mathrm{c}}$ an, dann ergibt der Erwartungswert des Zweikörperpotentials ${ }^{\mathbf{9}}$ :

$$
\begin{aligned}
<V^{2 K}>=\frac{1}{2} \sum_{i, j=1}^{A} \int \mathrm{dr}_{1} \mathrm{dr}_{2} \varphi_{i}^{*} & (1) \varphi_{j}^{*}(2) V(12) \\
\cdot & \left|\begin{array}{ll}
\varphi_{i}(1) \varphi_{i}(2) \\
\varphi_{j}(1) & \varphi_{j}(2)
\end{array}\right| f^{2}\left(r_{12}\right),
\end{aligned}
$$

wo $f(r)=1$ bzw. 0 für $r>$ bzw. $<r_{\mathrm{c}}$. Die Funktionen $\varphi_{i}(j)$ werden durch ebene Wellen im Kernvolumen $v$ approximiert.

Um den Erwartungswert des Potentials als Funktion der Kerndichte $\varrho$ zu schreiben, führen wir die Größe $\zeta=\varrho / \varrho_{0}$ ein, wobei wir als Bezugsdichte $\varrho_{0}$ die empirische Kerndichte $\varrho_{0}=\left(3 \mathrm{~m}^{3} / 4 \pi\right)$ wählen. Wird für den Maximalimpuls der Fermi-Kugel die Beziehung $k_{m}=1,52 \mathrm{~m} \zeta^{1 / 3}$ verwendet, so erhält man schließlich als Erwartungswert des Zweikörperpotentials pro Nukleon:

$$
\begin{aligned}
<V^{2 K}> & \mid A=(3 / 2) \zeta \int_{x_{\mathrm{c}}}^{\infty} \mathrm{d} x x^{2} V_{\mathrm{z}}^{2 K}(x)+\left(27 \zeta^{1 / 3} / 8 \cdot 1,\right. \\
& \cdot \int_{x_{\mathrm{c}}}^{\infty} \mathrm{d} x\left[-V_{\mathrm{z}}^{2 K}+3 V_{\mathrm{a}}^{2 K}\right]\left(j_{1}\left(1,52 \zeta^{1 / 3} x\right)\right]^{2},
\end{aligned}
$$

wobei das Zentral- und das Austauschpotential durch $V_{\mathrm{z}}^{2 K}=V_{4}^{\mathrm{a}}+V_{4}^{\mathrm{b}}+V_{4}^{\prime}$ und $V_{\mathrm{a}}^{2 K}=V_{2}+V_{6}$ für den ${ }^{1} S$-Zustand gegeben sind. Der zweite Summand in (34) stellt den Austauschterm dar. (34) gibt für $\left(f^{2} / 4 \pi\right)=14$ :

$$
<V^{2 K}>\mid A=-66,3 \mathrm{MeV} \zeta+a(\zeta)
$$

mit $a(1)=12,1 \mathrm{MeV}$. Der Austauscheffekt beträgt also $18 \%$ für $\zeta=1$ (Drell und Huang erhalten für ihr Potential $19 \%$ bei $\zeta=1$ ).

Die Erwartungswerte der $n$-Körperkräfte lassen sich für $n \geqq 3$ aufsummieren zu

$$
W(\zeta)=\sum_{n=3}^{\infty}<V^{n K}>/ A .
$$

Bei Vernachlässigung von Austausch- und Rückstoßeffekten erhalten Drell und Huang:

$$
\begin{aligned}
& W(\zeta)=(m / 16 \pi)\left[\left(1+\gamma^{2}\right) \ln (1+\gamma)-\gamma-1,5 \gamma^{2}\right] \\
& \text { für } \zeta \gg 1,
\end{aligned}
$$

wo $\gamma=6\left(f^{2} / 4 \pi\right)(m / 2 M) \zeta=6,3 \zeta$ für $\left(f^{2} / 4 \pi\right)=14$. 
(Die Vernachlässigung der Austauschterme ist für $\zeta \gg 1$ zulässig, da der Anteil der Austauschterme für große Dichten sehr klein ist.)

Die kinetische Energie pro Nukleon beträgt für Kerne, die in den Proton- und Neutronzahlen symmetrisch sind:

$$
E_{\mathrm{kin}} / A=E_{\mathrm{F}}\left(\zeta^{2 / 3}+0,82 \zeta\right),
$$

wo die Fermi-Energie $E_{\mathrm{F}}$ durch

$$
E_{\mathrm{F}}=\left(3 m^{3} / 40 M\right)(9 \pi)^{2 / 3}=14,7 \mathrm{MeV}
$$

gegeben ist.

Die Energie pro Nukleon ergibt sich durch Addition von (35), (38) und dem Mehrkörperpotential (36), das für $\zeta \gg 1$ durch (37) gegeben ist. (Es handelt sich dabei um die Volumsenergie pro Nukleon, da die Oberflächen- und Coulomb-Energie hier nicht in Betracht gezogen wurde.)

Zusammenfassend läßt sich folgendes sagen: Der starke Anstieg von (37) für $\zeta<1$ hindert den Kern am Zusammenstürzen. Ob die Energie pro Nukleon für $\zeta=1$ ein Minimum hat, läßt sich schwer entscheiden. Sollen (35), (36) und (38) die experimentelle Volumsenergie pro Nukleon richtig wiedergeben, dann müssen für $\zeta=1$ die Austausch- und Nukleonenrückstoßeffekte den Beitrag von (37) auf $16 \%$ seines ,,klassischen“"Wertes reduzieren. Das ist ein Wert, der nach den Berechnungen von D r ell und Huang über die Austauscheffekte bei der Drei-, Vier- und Fünfkörperkraft und nach den Berechnungen in dieser Arbeit über Nukleonenrückstoßeffekte vernünftig erscheint.

\section{Spin-Bahn-Kopplung}

Einen Spin-Bahn-Beitrag erhält man von Übergängen, bei welchen ein Zwischenzustand negativer
Energie durchlaufen wird. Dadurch ergeben sich Übergangselemente folgender Art:

$$
\begin{aligned}
<\mathfrak{p}\left|\beta \gamma_{5} \Lambda\left(\mathfrak{p}-\mathfrak{f}_{1}\right) \beta \gamma_{5}\right| \mathfrak{p}-\mathfrak{f}_{1}-\mathfrak{f}_{2}> \\
=1+(2 M)^{-2} \vec{\sigma} \cdot i\left(\mathfrak{f}_{1}+\mathfrak{f}_{2}\right) \times \mathfrak{p} .
\end{aligned}
$$

Das Potential zweiter Ordnung enthält keine solchen Terme. Bei den Beiträgen vierter Ordnung kommen sie in den Zwei- und Einpaartermen vor. Die Berücksichtigung von Spin-Bahn-Beiträgen bedeutet also, daß wir die Integranden von $V_{4}^{\mathrm{a}}, V_{4}^{\mathrm{b}}$ und $V_{4}^{\prime}$ mit (39) zu multiplizieren haben. Die Berücksichtigung des zweiten Termes von (9) liefert:

$$
V_{4} \psi \rightarrow V_{4} \psi+(2 M)^{-2} \operatorname{grad} V_{\mathrm{Sp} . \mathrm{B} .}^{4} \times \mathfrak{p} \cdot \vec{\sigma} \psi,
$$

wo $V_{4}=V_{4}^{\mathrm{a}}+V_{4}^{\mathrm{b}}+V_{4}^{\prime}, V_{\mathrm{Sp} \text {. B. }}^{4}=2\left(V_{4}^{\mathrm{a}}+V_{4}^{\mathrm{b}}\right)+V_{4}{ }^{\prime}$.

Wir erhalten also einen Spin-Bahn-Beitrag

$$
\left(4 M^{2} r\right)^{-1}(\mathrm{~d} / \mathrm{d} r) V_{\mathrm{Sp} . \mathrm{B} .}^{4}\left(\overrightarrow{l_{\mathrm{a}}} \cdot \overrightarrow{S_{\mathrm{a}}}+\overrightarrow{l_{\mathrm{b}}} \overrightarrow{S_{\mathrm{b}}}\right),
$$

wo $\vec{S}=1 / 2 \vec{\sigma}, \vec{l}=\mathfrak{r} \times \mathfrak{p}$. Da nun $V_{\mathrm{Sp} . \text { B. }}^{4}$ mit $r$ monoton wächst, liefert das Potential vierter Ordnung einen Spin-Bahn-Beitrag mit positivem Vorzeichen,

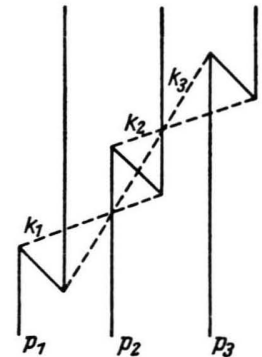

Abb. 10. Graph zur Dreikörperkraft. das jedoch den Ergebnissen der Kernspektroskopie widerspricht. Kommt der Ausdruck (39) in einem anziehenden bzw. abstoßenden Potential vor, so erhält der Spin-Bahn-Beitrag ein positives bzw. negatives Vorzeichen. Ein abstoßendes Potential liegt aber im Falle der Dreikörperkraft vor, die nach den Überlegungen des vorangehenden Abschnittes nicht zu vernachlässigen ist. Der Hauptterm (vgl. Abb. 10) enthält nur Übergänge der gewünschten Form:

$$
\begin{gathered}
V^{3 K}\left(r_{12}, r_{23}, r_{13}\right)=\left(f^{2} / 2 M\right)^{3} 6(2 \pi)^{-9} \int \mathrm{d}^{3} \mathfrak{f}_{1} \mathrm{~d}^{3} \mathfrak{f}_{2} \mathrm{~d}^{3} \mathfrak{f}_{3}\left(\omega_{1} \omega_{2} \omega_{3}\right)^{-1}\left\{\left[\left(\omega_{1}+\omega_{3}\right)\left(\omega_{2}+\omega_{3}\right)\right]^{-1}+\mathrm{zykl} .\right\} \cdot \\
\cdot M_{1} M_{2} M_{3} \exp \left(i \mathfrak{f}_{1} \mathrm{r}_{12}+\mathrm{zykl} .\right),
\end{gathered}
$$

wo $M_{1}=\left\langle\mathfrak{p}_{1}\left|\beta \gamma_{5} \Lambda_{-}\left(\mathfrak{p}_{1}-\mathfrak{f}_{1}\right) \beta \gamma_{5}\right| \mathfrak{p}_{1}-\mathfrak{f}_{1}+\mathfrak{f}_{3}>=1+i(2 M)^{-2}\left(\left[\mathfrak{f}_{1}-\mathfrak{f}_{3}, \mathfrak{p}_{1}\right] \cdot \vec{\sigma}_{1}\right)+\ldots\right.$, zykl.

Vernachlässigen wir Beiträge $\sim(2 M)^{-7}$, so gibt $(42): V_{\mathrm{z}}^{3 K}+(2 M)^{-2}\left\{\left[\left(\nabla_{12}+\nabla_{13}\right) V_{\mathrm{z}}^{3 K}, \mathfrak{p}_{1}\right] \cdot \vec{\sigma}_{1}+\mathrm{zykl}\right.$. $\}$,

$$
\text { wo } V_{\mathrm{z}}^{3 K}\left(x_{12}, x_{23}, x_{13}\right)=12\left(f^{2} / 4 \pi\right)^{3}(m / 2 M)^{3} m(2 / \pi) K_{1}\left(x_{12}+x_{23}+x_{13}\right) /\left(x_{12} x_{23} x_{13}\right) \text {. }
$$

Um etwas über die Größe dieses Spin-Bahn-Beitrages zu erfahren, betrachten wir den Erwartungswert der Spin-Bahn-Wechselwirkungsenergie eines Nukleons (Index $k$ ) mit einem g-g-Rumpf:

$$
<V_{\text {Sp. в. }}^{3 K}>=(1 / 6) \sum_{i, j=1}^{A} \int \mathrm{dr}_{1} \mathrm{dr}_{2} \mathrm{dr}_{3} \varphi_{i}^{*}(1) \varphi_{j}^{*}(2) \varphi_{k}^{*}(3) V_{\mathrm{Sp.} \mathrm{B.}}^{3 K} \text { Det }\left|\varphi_{l}(m)\right| f^{2},
$$


wo $f=f\left(r_{12}\right) f\left(r_{\varepsilon_{3}}\right) f\left(r_{13}\right), f(r)=0$ bzw. 1 für $m r<$ bzw. $>0,38$. Bei Vernachlässigung von Rückstoß- und Austauscheffekten erhalten wir mit

$V_{\mathrm{Sp.} \mathrm{в.}}^{3 K}=(24 / \pi)\left(f^{2} / 4 \pi\right)^{3}(m / 2 M)^{5} m\left\{\left[x_{12}{ }^{-1}\left(\mathrm{~d} / \mathrm{d} x_{12}\right)\left(K_{1}\left(x_{12}+x_{23}+x_{13}\right) / x_{12} x_{23} x_{13}\right) \mathfrak{x}_{12}+2 \rightleftarrows 3, \mathfrak{p}_{1}\right] \cdot \vec{\sigma}_{1}+\right.$ zykl. $\}:$

$<V_{\mathrm{Sp.} \mathrm{B.}}^{3 K}>=-(1 / 6)\left(\varrho / m^{3}\right)^{2} 32 \pi^{2}(24 / \pi)\left(f^{2} / 4 \pi\right)^{3}(m / 2 M)^{5} m$

$$
\cdot \int_{x_{\mathrm{c}}}^{\infty} \mathrm{d} x \int_{x_{\mathrm{c}}}^{\infty} \mathrm{d} y\left|K_{1}(x+y+z) / z\right|_{z=\max \left(x_{\mathrm{c}},|x-y|\right)}^{x+y} .
$$

Dies gibt mit $\quad x_{\mathrm{c}}=0,38$ und $\left(f^{2} / 4 \pi\right)=14:<V_{\mathrm{Sp} . \mathrm{B} .}^{3 K}>=-7,8 \mathrm{MeV} \zeta^{2}\left(\vec{l}_{\mathrm{k}}, \vec{S}_{\mathrm{k}}\right)$,

während der entsprechende Ausdruck für das Zweikörperpotential lautet:

$$
<V_{\mathrm{Sp.} \mathrm{B.}}^{2 K}>=1,5 \zeta\left(\vec{l}_{\mathrm{k}}, \vec{S}_{\mathrm{k}}\right) \int_{x_{\mathrm{c}}}^{\infty} \mathrm{d} x x^{2}(m / 2 M)^{2}(\mathrm{l} / x)(\mathrm{d} / \mathrm{d} x) V_{\mathrm{Sp} . \text { B. }}^{4}=9,8 \operatorname{MeV} \zeta\left(\vec{l}_{\mathrm{k}}, \vec{S}_{\mathrm{k}}\right) .
$$

Der Erwartungswert der Austauschterme wurde nicht berechnet. Es sollte nur gezeigt werden, daß die Dreikörperkraft einen Spin-Bahn-Beitrag mit dem richtigen Vorzeichen und in einer vernünftigen Größenordnung liefert. Es könnte also möglicherweise die Summation über alle Mehrkörperkräfte ungefähr zur richtigen Spin-Bahn-Kopplung füh- ren, ohne daß man dafür die schwereren Mesonen heranziehen müßte.

Herrn Professor Heisenberg danke ich für sein förderndes Interesse und der Deutschen Forschungsgemeinschaft für die Ermöglichung meines Göttinger Aufenthaltes.

\title{
Nichtlokale Feldtheorie auf der Grundlage der Salpeter-Bethe-Gleichung
}

\section{Wechselwirkung mit lokalisiertem Teilchen}

\author{
Von H. L. Jordan und W. E. Frahn \\ Aus dem Institut für theoretische Physik und dem Physikalischen Institut der Tech- \\ nischen Hochschule Aachen \\ (Z. Naturforschg. 9 a, 572-578 [1954]; eingegangen am 23. April 1954)
}

Im Anschluß an eine vorhergehende Arbeit $^{1}$ wird die Salpeter-Bethe-Gleichung für den Fall der Wechselwirkung mit einem lokalisierten schweren Teilchen behandelt. Es wird gezeigt, daß die Wechselwirkung bereits in zweiter störungstheoretischer Näherung nichtlokale Struktur besitzt. Am Beispiel des einfachsten irreduziblen Graphen vierter Ordnung wird die Wechselwirkungsfunktion explizit berechnet, ihre Raumzeitstruktur und der Übergang zu lokalen Potentialen in adiabatischer Näherung diskutiert.

$\mathrm{I}$ n I wurde aus der Zweiteilchen-Salpeter-BetheGleichung durch Reduktion auf das Einteilchenproblem eine verallgemeinerte Dirac-Gleichung gewonnen, welche die Bewegung eines ,freien“ Teilchens bei Wechselwirkung mit einem nirgends lokalisierten und daher durch ebene Wellen darstellbaren Feld beschreibt. Die erhaltene Gleichung hat die Form der aus nichtlokalen Theorien freier Teilchen bekannten Verallgemeinerungen der Dirac-Gleichung.

${ }^{1}$ H. L. Jordan u. W. E. Frahn, Z. Naturforschg. 8a, 620 [1953], im folgenden mit I bezeichnet.
In der vorliegenden Arbeit untersuchen wir in entsprechender Weise den Fall der Wechselwirkung eines Teilchens mit einem räumlich lokalisierten schweren Teilchen. Dabei zeigt sich, daß die Wechselwirkung nur in niedrigster Ordnung der Störungsrechnung (Leiter-Approximation) durch ein lokales Potential beschrieben wird, während die Terme höherer Ordnung nichtlokale Potentiale ergeben. Nach der Reduktion der Salpeter-BetheGleichung (SBG) auf den Einteilchenfall (§ 1) werden die von einem lokalisierten schweren Teilchen herrührenden Wechselwirkungsanteile niedrigster Ordnung berechnet (§ 2) und die Raum-Zeit-Struktur 